\title{
Entrelacs
}

ENTRELACS Cinéma et audiovisuel

18 | 2021

Laurent Roth, cinéaste

\section{Les Yeux brûlés, un astre dans le temps}

Julie Savelli

\section{(2) OpenEdition}

Journals

Édition électronique

URL : https://journals.openedition.org/entrelacs/6148

DOI : 10.4000/entrelacs.6148

ISSN : 2261-5482

Éditeur

Éditions Téraèdre

\section{Référence électronique}

Julie Savelli, «Les Yeux brûlés, un astre dans le temps », Entrelacs [En ligne], 18| 2021, mis en ligne le

13 décembre 2021, consulté le 15 décembre 2021. URL : http://journals.openedition.org/entrelacs/

$6148 ;$ DOI : https://doi.org/10.4000/entrelacs.6148

Ce document a été généré automatiquement le 15 décembre 2021.

Tous droits réservés 


\title{
Les Yeux brûlés, un astre dans le temps ${ }^{1}$
}

\author{
Julie Savelli
}

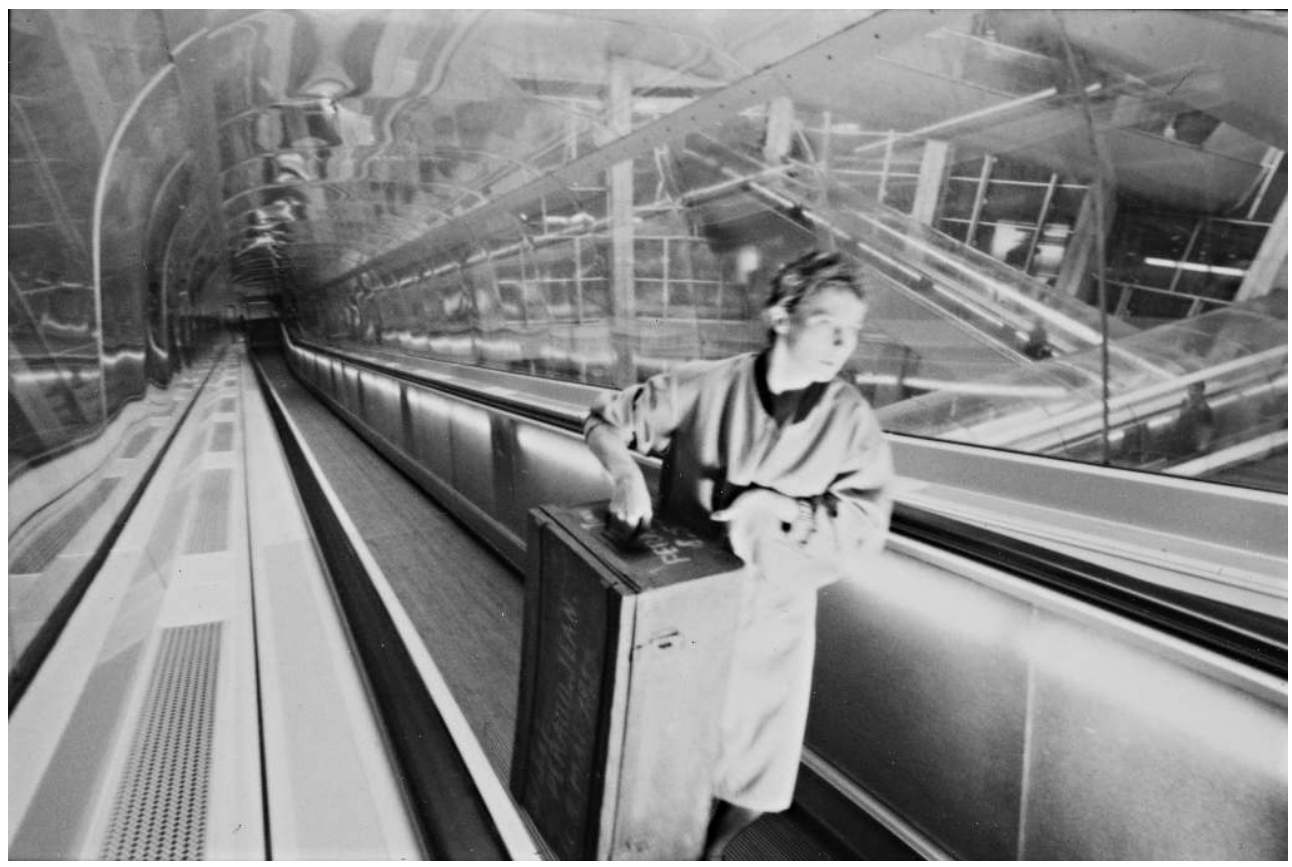

Mireille Perrier et « la cantine du rapatrié ».

$1 \quad$ Les Yeux brûlés est un film miraculé qui aura rayonné tel un astre pendant près de 30 ans dans les fonds de l'Établissement Cinématographique et Photographique des Armées (l'ECPAD) avant d'être restauré par le CNC et de sortir en salles le 11 novembre 2015 avec les honneurs du festival de Cannes (sélection Cannes-Classics). Son auteur, Laurent Roth, fait ses débuts en cinéma quand il réalise ce film en 1986 dans le cadre d'une commande passée par le Service Cinéma de l'Armée. Le jeune appelé, philosophe de formation, plonge alors dans les archives (14-18, Indochine, Algérie) et s'attache à mettre le spectacle de la guerre en lumière par les moyens du cinéma. Il élabore un huis 
clos fictionnel de type SF, utilisant le hall d'attente de Roissy comme un studio-matrice en couleurs. Cet étrange protocole de visibilité va permettre au spectre noir et blanc de l'action militaire de se redéployer sous nos yeux. Et ce sont alors différents régimes d'images (scènes jouées, journaux d'actualité, photographies et films de guerre) qui s'entremêlent sur un mode exploratoire pour tisser le patchwork d'un récit qui œuvre à la métamorphose document-documentaire-fiction.

Les Yeux brûlés s'inscrit dans la filiation d'un cinéma résolument moderne. Aéroport Charles de Gaulle, intérieur jour : on pense à la mythique Jetée de Chris Marker (1962) en partie tournée sur la terrasse de l'aérogare d'Orly. Une jeune comédienne joue son propre rôle ; il s'agit de Mireille Perrier. Révélée peu de temps auparavant, elle vient de tourner Boy Meets Girls de Leos Carax (1984) et Elle a passé tant d'heures sous les sunlights... de Philippe Garrel (1985). Suivie par un homme dont la profession est celle de reporter, nous observons aujourd'hui avec une merveilleuse distance cinéphilique cette " altra ego » du cinéaste, selon l'expression de Serge Daney dans Libération ${ }^{2}$. La jeune femme est venue réceptionner la "cantine du rapatrié ». Celle-ci contient les effets et les clichés du photo-reporter Jean Peraud, disparu à Diện Biên Phu le 8 mai 1954. Six "soldats de l'image " + 1 vont se succéder autour de la malle fantôme de leur compagnon. André Lebon, Daniel Camus, Pierre Ferrari, Raoul Coutard, Marc Flament et Pierre Schoendoerffer s'entretiennent avec Mireille Perrier à l'écran sans texte ni dialogues écrits, tandis que Raymond Depardon intervient ponctuellement en off telle la voix aveugle du récit.

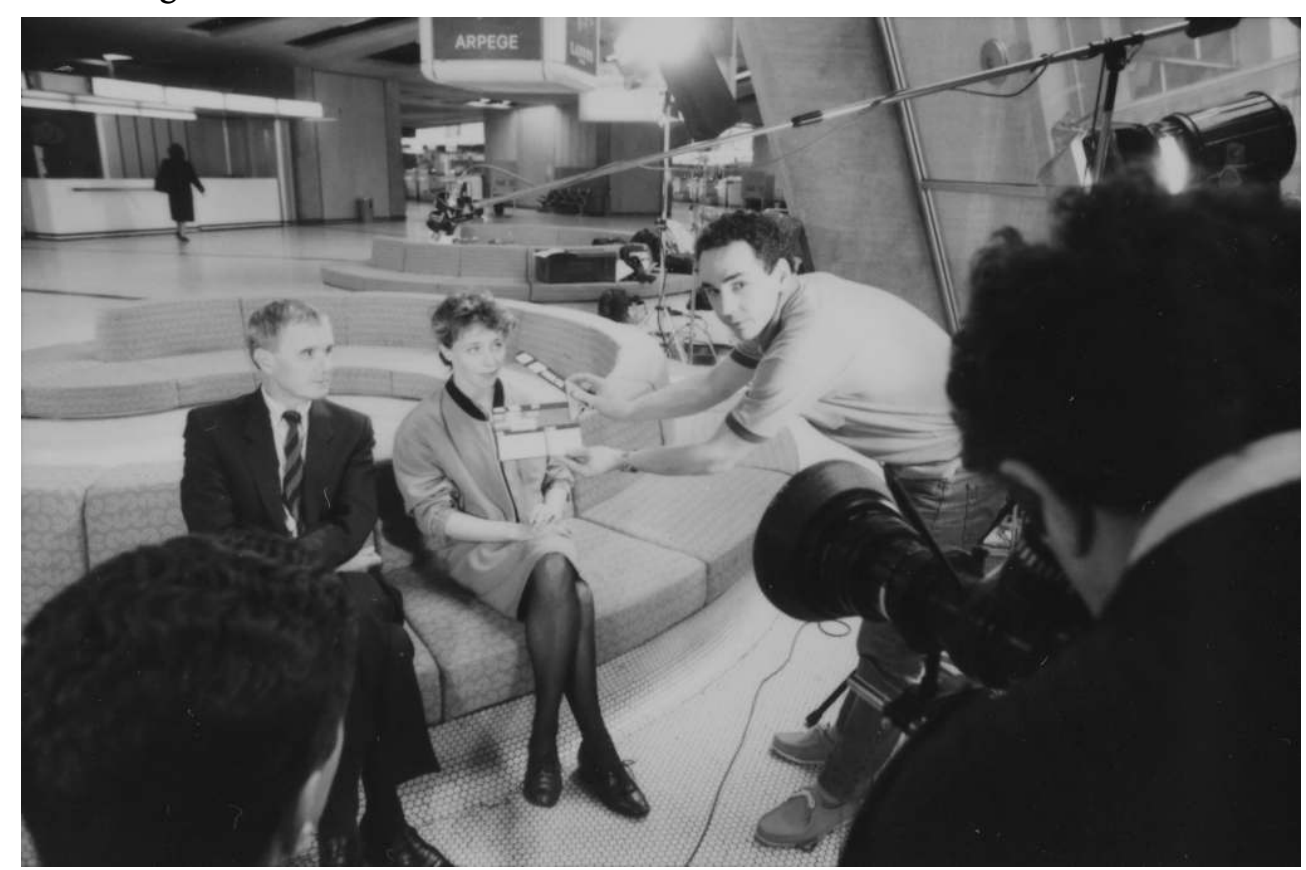

Pierre Schoendoerffer, Mireille Perrier, Hervé Lavergne au clap. 


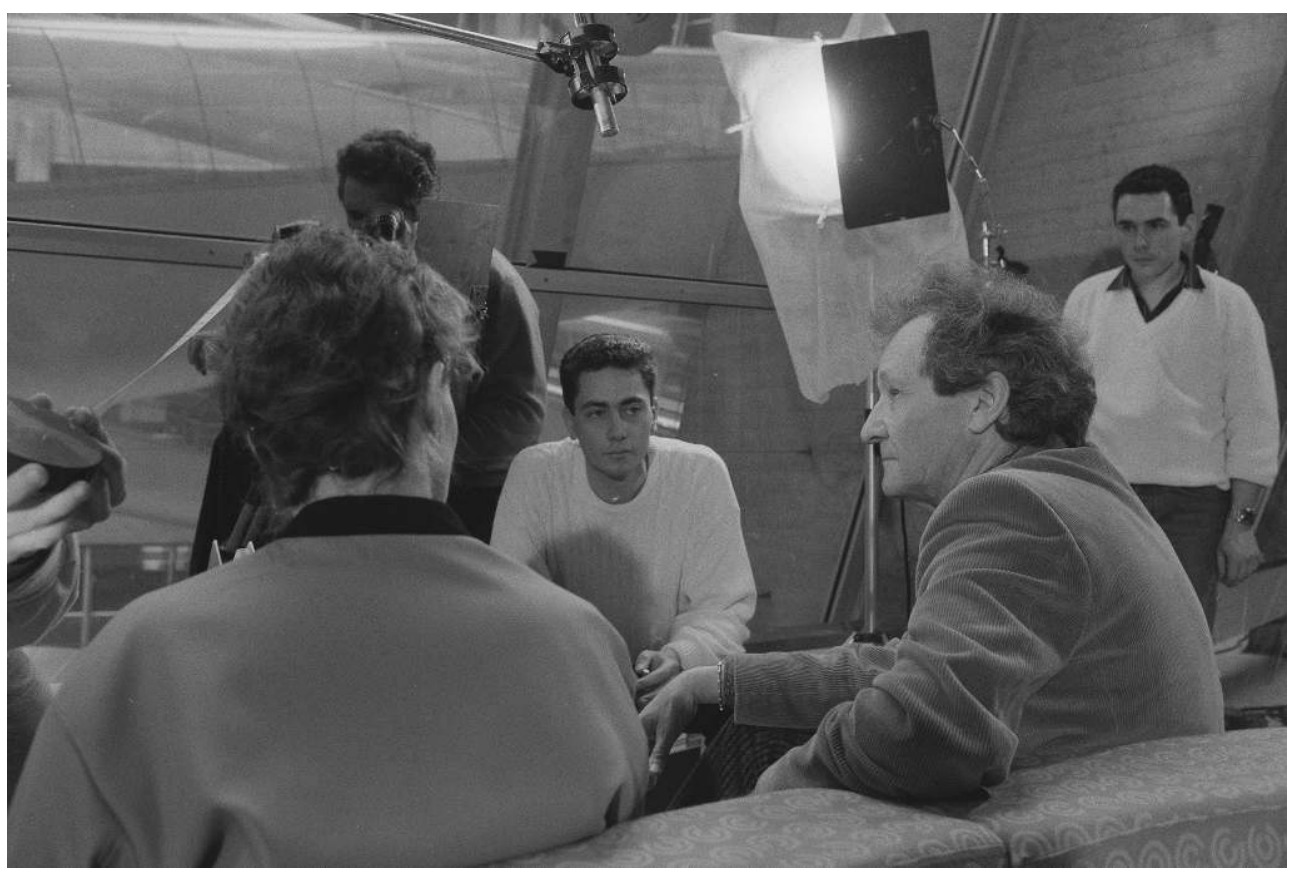

De gauche à droite : Mireille Perrier, Bernard Miale (chef-opérateur), Laurent Roth, Marc Flament, Hervé Lavergne (électricien).

3 Si la fiction se prête dès lors à une lecture documentarisante, ses procédés participent aussi d'un fantasme et placent le témoignage des reporters dans un "ailleurs" superbement incarné par la féminité de Mireille Perrier. La parole masculine est suscitée puis relancée par la spontanéité et l'émotion de la jeune comédienne qui improvise sans filets. Pourquoi filmer la machine de la guerre ? Et pour produire quoi : de la beauté, du spectacle, un « document humain »? Cette image est-elle la résultante de la peur de la mort ou du goût que le reporter a pour elle? Autant de questions, parmi beaucoup d'autres, que (se) pose Mireille Perrier pour tenter de réfléchir la représentation de la guerre. «On peut couper?» demande Marc Flament, visiblement éprouvé par la détermination de la jeune femme dont la pâleur ne trompe pas... Pour ces entretiens, la méthode est empruntée à Lanzmann.

Il fallait armer Mireille, écrit Laurent Roth dans le dossier de presse du film. [...] La méthode Lanzmann consistait à doucement insister et déplacer : insister sur les questions, les reposer plusieurs fois, différemment ; déplacer le discours construit, en partant sur des émotions, non dites. 


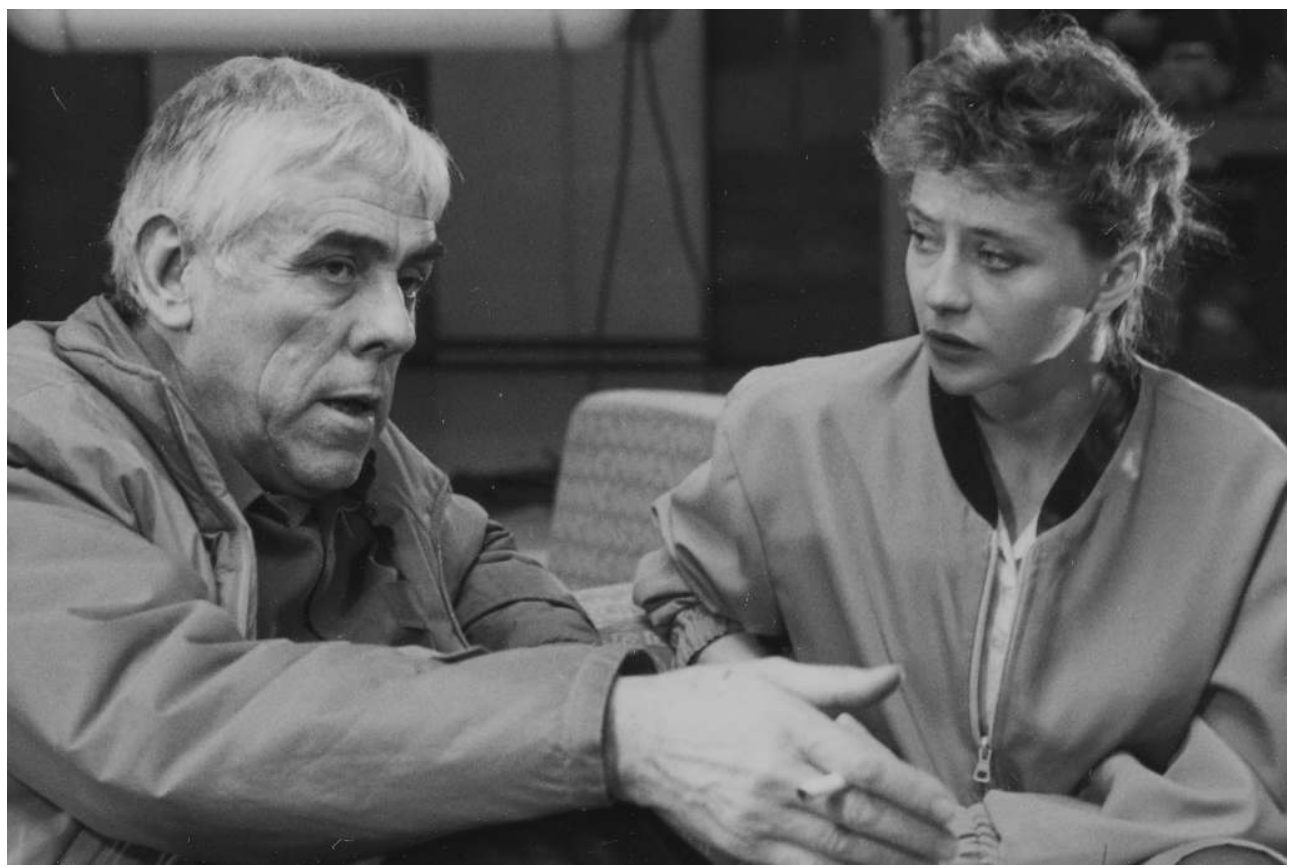

Raoul Coutard et Mireille Perrier.

De sorte que les mots doublent rarement l'image; ils la creusent cherchant plutôt à démasquer la sensibilité refoulée pour révéler une fissure intérieure. Et Serge Daney de conclure sa critique du film par le constat d'une commande non pas simplement détournée, mais «retournée au commanditaire, avec accusé réception». Car la question de la réception est bien au cœur de ce film dont la séquence de fin, sublime, incarne tout l'état d'être. Mireille Perrier est-elle dirigée quand, assise dans un taxi qui file sur le périphérique, perlent à ses joues les larmes que le film contient en creux ? Il est fort à parier que oui, à moins que... non ${ }^{3}$. Peu importe car si la comédienne pense à vue, elle pleure ici en son nom, exprimant toute la violence intérieure contenue par le dispositif performatif de la mise en scène.

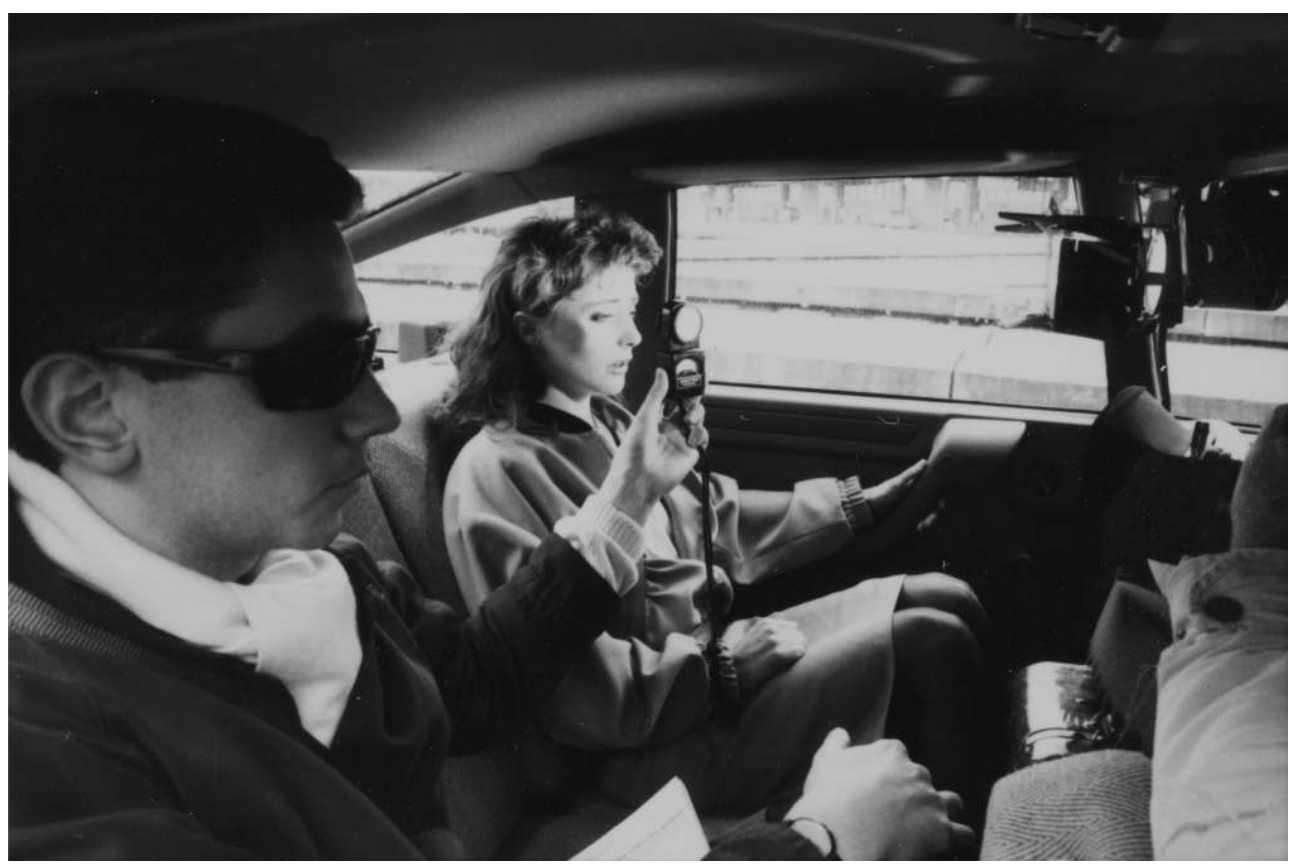

Laurent Roth, Mireille Perrier (réglage du plan de fin). 
Ce film rescapé n'est pas simplement catapulté, près de trente ans plus tard, dans notre actualité cinématographique; son sujet continue de brûler aujourd'hui. L'image de guerre, professionnelle et amateur, habite plus que jamais nos écrans (fixes et mobiles) alors même que nous manquons souvent de recul quant à la mise en scène des conflits. Sans doute est-il important d'informer et de s'informer, de donner à voir les images de notre temps, présent ou passé, mais ne faut-il pas aussi les faire parler, les re-tourner, afin de réfléchir le monde avec une nécessaire distance critique? C'est précisément ce que Laurent Roth réalise avec beaucoup de justesse dans Les Yeux brûlés. L'archive de guerre y est en prise comme rarement avec le cinéma, cherchant à savoir de quelle histoire nous sommes les héritiers, ou les acteurs, en même temps que les passeurs.

Crédit images: Patrice George - ECPAD.

\section{NOTES}

1. Cette critique a été publiée dans la revue Bref, le magazine du court métrage ( $\mathrm{n}^{\circ} 117,2015$, pp. 12-13) en 2015 lors de la sortie du film en salles, et légèrement revue pour sa reprise ici.

2. Serge Daney, Les Yeux brûlés, ou la métaphysique guerrière, Libération, 10 oct. 1986. Repris dans : La Maison cinéma et le monde tome 3 : les années Libé (1986-1991), P.O.L., 2012.

3. L'interrogation demeure dans le récent projet de Laurent Roth qui, trente ans après cette première collaboration, offre à nouveau un terrain de figuration libre à Mireille Perrier. La Joie, le film (2015) est l'adaptation cinématographique du second volet d'une trilogie écrite par Laurent Roth pour le théâtre, La Chose, La Joie, L'Âme. Filmée au théâtre du Rond-Point sans décors ni costumes, Mireille Perrier lit aux côtés de Mathieu Amalric, hésite parfois, reprend un mot tandis que le processus d'interprétation s'incarne sous nos yeux médusés.

\section{RÉSUMÉS}

Hommage aux opérateurs de guerre, Les Yeux brûlés (1986) de Laurent Roth aurait pu rester pour toujours dans les archives audiovisuelles des armées si le distributeur Shellac ne s'était pas attelé à en renégocier les droits. La célébration, durant l'année 2015, du centenaire de la création des sections cinématographique et photographique des armées a heureusement facilité l'entreprise de son exhumation.

A tribute to war operators, Laurent Roth's Les Yeux brûlés (1986) could have remained forever in the audiovisual archives of the armed forces if the distributor shellac had not undertaken to renegotiate the rights. Fortunately, the celebration in 2015 of the centenary of the creation of the cinematographic and photographic sections of the armed forces facilitated the exhumation of the film. 


\section{AUTEUR}

\section{JULIE SAVELLI}

Maîtresse de conférences en études cinématographiques et audiovisuelles à l'université PaulValéry Montpellier 3 et membre du Centre de recherches RiIRRa21. Dans ses travaux qui portent sur la création documentaire contemporaine, elle s'intéresse plus particulièrement au cinéma engagé, à l'autobiographie en images et à l'anthropologie du visuel. Parmi ses diverses publications, mentionnons la direction de trois ouvrages collectifs en 2021 : Jean Rouch. Passeur d'images, passeur de mondes (Téraèdre), John Cassavetes. Imaginaires des corps, entre la scène et l'écran (PUP) et Dominique Cabrera. L'intime et le Politique (De l'Incidence Éditeur). 